\title{
The shielding of a microdisc electrode surrounded by an adsorbing surface
}

\author{
Shaltiel Elul Richard G. Compton*
}

\section{Abstract}

Microdisc electrodes are usually made by surrounding a metal wire of a diameter of a few microns in a flat insulating sheath made of glass or other insulating material. The sheath is generally assumed to play no role in the voltammetry observed at the microdisc electrode. However, in some cases, and especially for experiments with nano-particles, significant adsorption can occur on the sheath so perturbing the current response measured at the disc. We report theoretical calculations of the effect of 'shielding' by the insulating sheath of micro electrodes and show that this can significantly influence the magnitude of the current even for relatively small levels of adsorption, not least because of the typically very large area of the insulation relative to the electrode size. Working curves are provided to permit analysis of experimental data.

\section{Introduction}

Microelectrodes have transformed the dynamic range of electrochemistry since their introduction and popularization dating from the 1970s and 1980s $[1,2,3$, 4]. The diversity and range of their application continue to expand beyond that of the fundamental voltammetry research aimed at molecular solution phase species as originally pioneered. In particular, recent applications have included the addressing of biological problems $[5,6]$, the examination of surfaces with SCEM [7], covalently modified and functionalized microelectrodes for various applications[8], and the study of nano particles [9].

Microelectrodes are typically fabricated by insulating a metal wire of a micron diameter by a sheath made of glass or some other electrically non conducting metal [10]. The end of the structure is polished flat in practical use so as to expose a metal microdisc surrounded by a concentric ring of insulation. The latter is usually of the millimetre size. In analysing the current response seen at the microdisc electrode, it is usually assumed that the insulating sheath plays no

\footnotetext{
${ }^{*}$ Correspoder Author, Department of Chemistry, Physical and Theoretical Chemistry Laboratory, Oxford University, South Parks Road, Oxford OX1 3QZ, United Kingdom
} 
role other than to provide a non-conducting surround and at which no material fluxes arrive or leave. This is undoubtedly an excellent assumption for molecular voltammetry conducted in conventional media. However, in the context of the study of biological media, functionalized surfaces, or nano-particles, this may no longer be the case. In particular, if the species of interest (by virtue of their voltammetry at the microdisc surface) adsorb on the insulation sheath, then, because of the large relative area of this potential material 'sink', a significant perturbation of the microdisc current can arise. Such considerations may play an important role in the area of 'nano-impacts' where the microelectrode preparation is extremely important for the quantitative analysis of nano-particles and some evidence of 'shielding' has been noted[11, 12].

In this paper, we report simulations that quantify the effect of shielding caused by adsorption on the insulating sheath of the microelectrodes, and demonstrate that this can have a very significant influence on the currents measured at microdisc electrodes.

\section{Theory}

We develop a model to simulate the shielding effect on the flux of electroactive species, towards a microdisc electrode surrounded by an insulating but sticking surface. This study is made via simulating Fickian diffusion under chrono-amperomtery in the presence of supporting electrolyte to assume diffusion controlled transport. By approximating the transport to behave in a statistical manner[13, 14, 15, 16], we can avoid complex stochastic modelling and solve numerically Fick's second law [17]. For the microdisc geometry, we solve Fick's second law for cylindrical space using axial symmetry around $r=0$ [18]:

$$
\frac{\partial c}{\partial t}=D\left(\frac{\partial^{2} c}{\partial r^{2}}+\frac{\partial^{2} c}{\partial z^{2}}+\frac{1}{r} \frac{\partial c}{\partial r}\right)
$$

where $D$ is the diffusion coefficient, $c$ is the concentration, and $r$ and $z$ are cylindrical coordinates. In this space, the axis $z$ passes through the centre of the disc and is perpendicular to its plane. Therefore, we define two distances in the $r$ direction, the disc electrode $\operatorname{radius}\left(r_{d}\right)$ and the surface radius $\left(r_{s}\right)$ as illustrated in figure 1.

Dimensionless parameters are used in the simulation to simplify the analysis [18]. Coordinates are normalized to the surface radius $\left(R=r / r_{s}, Z=z / r_{s}\right)$ and concentrations are defined relative to the bulk concentration of the electroactive species $\left(C=c / c^{*}\right)$. A dimensionless time parameter $(\tau)$ is defined as:

$$
\tau=\frac{D}{r_{s}^{2}} t
$$

All the dimension and dimensionless symbols are summarized in Table 1. In the transformed coordinates the radius of the surface is unity and the microdisc lies 
between: $0<R_{d}<1$. Converting Fick's second law into a dimensionless form gives[19]:

$$
\frac{\partial C}{\partial \tau}=\left(\frac{\partial^{2} C}{\partial R^{2}}+\frac{\partial^{2} C}{\partial Z^{2}}+\frac{1}{R} \frac{\partial C}{\partial R}\right)
$$

The dimensionless mass transport equation must be solved over all space with the appropriate boundary conditions which define the kinetics and fluxes on the surfaces of the simulation space.

\subsection{Boundary Conditions}

At $z=0$ we define the kinetics of the sticking surface and the electrode boundary condition. At the disc electrode, the concentration is set to zero, assuming mass transport control:

$$
\text { at } \quad \tau \geq 0 \text { and } R \leq R_{d}, C_{R, 0}=0
$$

We next consider the boundary condition for the sticking surface[20, 13]. The concentration adjacent to the surface drops due to adsorption. This boundary condition is assumed to be a first order kinetic irreversible adsorption according to the Langmuirian model[21, 20]. Therefore, the flux on the sticking surface is given by:

$$
D\left(\frac{\partial c}{\partial z}\right)_{z=0}=k_{0}(1-\theta) c_{r, 0}
$$

where $(\theta)$ is the fractional coverage and varies between $0-1$. The adsorption rate constant $\left(\mathrm{cm} \mathrm{s}^{-1}\right)$ is denoted by $k_{0}$. The rate constant $k_{0}$ can be related to a 'sticking coefficient' which is experimentally accessible in some cases e.g. [22]. The relationship is discussed in the Appendix to this paper. Converting $k_{0}$ to a dimensionless rate constant gives $K$ :

$$
K=\frac{k_{0} r_{s}}{D}
$$

Rewriting the flux equation in a dimensionless form gives the surface boundary condition:

$$
\begin{aligned}
& \tau \geq 0 \\
& R_{d}<R \leq R_{s} \quad\left(\frac{\partial C}{\partial Z}\right)_{Z=0}=K(1-\theta) C_{R, 0}
\end{aligned}
$$

The surface coverage $\left(\Gamma /\right.$ mole $\left.\mathrm{cm}^{-2}\right)$ is defined as the density of the maximum coverage. Hence, the variation of $\theta$ with $t$ can be defined as:

$$
\Gamma \frac{\partial \theta}{\partial t}=k_{0}(1-\theta) c_{r, 0}
$$

Converting into dimensionless form gives:

$$
\frac{\partial \theta}{\partial \tau}=K(1-\theta) \frac{C_{R, 0}}{\gamma}
$$


where $\gamma$ is a dimensionless coverage defined by:

$$
\gamma=\Gamma /\left(c^{*} D / k_{0}\right)
$$

Beyond the sticking surface, at $Z=0$, an insulation boundary condition is applied:

$$
R_{s}<R \leq R_{\max } \quad\left(\frac{\partial C}{\partial Z}\right)_{Z=0}=0
$$

The edges in the outer space of the simulation set to the bulk concentration:

$$
C_{R_{\max }}=1, \quad C_{Z_{\max }}=1
$$

where $R_{\max }$ and $Z_{\max }$ are set to be far enough from the root mean squared displacement according to the Einstein equation [23, 24]:

$$
\begin{aligned}
R_{\max } & =1+6 \sqrt{\tau_{\max }} \\
Z_{\max } & =6 \sqrt{\tau_{\max }}
\end{aligned}
$$

$\tau_{\max }$ is chosen to be sufficiently large to achieve a full convergence of the flux in chronoamperometry. From symmetry, the flux across the $Z$ axisymmetric boundary can be set to zero:

$$
\left(\frac{\partial C}{\partial R}\right)_{R=0}=0
$$

The dimensionless flux inward the disk or the electrode is given by:

$$
\begin{array}{ll}
\text { disk : } & 0<R \leq R_{d} \\
\text { surface }: & R_{d}<R \leq R_{s}
\end{array} \quad j=\left(\frac{\partial C}{\partial Z}\right)_{Z=0}
$$

By integrating the flux $\left(j^{D}\right)$ over the disc and the surface, we can obtain the net $\operatorname{flux}\left(J^{D}\right)$ inward the disc electrode and the adsorbing flux to the surface, respectively:

$$
J_{d}^{D}=2 \pi \int_{0}^{r_{d}} j_{r, 0}^{D} r \mathrm{~d} r, \quad J_{s}^{D}=2 \pi \int_{r_{d}}^{r_{s}} j_{r, 0}^{D} r \mathrm{~d} r
$$

In dimensionless form we get:

$$
J_{d}=2 \pi \int_{0}^{R_{d}} j_{R, 0} R \mathrm{~d} R, \quad J_{s}=2 \pi \int_{R_{d}}^{R_{s}} j_{R, 0} R \mathrm{~d} R
$$

Assuming a single electron transfer, the dimensional Faradaic current inward the microdisc electrode is obtained as follows:

$$
I_{d}=2 \pi F c^{*} D r_{s} \int_{0}^{R_{d}} j_{R, 0} R \mathrm{~d} R,
$$

The shielding effect then can be derived and compared to analytical calculations of a full sticking or non-sticking surface at different radius ratios, $R_{s d}$ :

$$
R_{s d}=\frac{R_{s}}{R_{d}}
$$




\subsection{Numerical Methods}

We use the finite difference approximation with the Crank-Nicolson method[25] to solve a set of concentration equations simultaneously over the $2 \mathrm{D}$ space at each point in time. These equations are discritized over an expanding spatial 2D grid with $m$ rows and $n$ columns $(R(n) \times Z(m))$ and dimensionless time $1 \mathrm{D}$ grid $(\tau(k))$. The spatial grid is generated over the space with 3 nodes $\left(N_{1-3}\right)$ at: $\delta R, R_{d}, R_{s}$. The neighbours points around these nodes are set to be with the smallest differences $\delta R$ and $\delta Z$, after which the grid differences grow exponentially to achieve full cover of all the space according to the following definitions:

$$
\begin{aligned}
& R \text { grid: } \\
& N_{1}=\delta R, \quad N_{2}=R_{d}, N_{3}=R_{s}, \\
& R_{n+1}=R_{n} \gamma_{R} \quad N_{j}<R_{n}<\frac{N_{j}+N_{j+1}}{2}, R_{s}<R_{n}<R_{\text {max }} \\
& R_{n+1}=R_{n} / \gamma_{R} \quad \frac{N_{j}+N_{j+1}}{2}<R_{n}<N_{j+1} \\
& Z \text { grid: } \\
& Z_{m+1}=Z_{m} \gamma_{Z} \quad 0<Z_{m}<Z_{\text {max }}
\end{aligned}
$$

where $\gamma_{R}$ and $\gamma_{Z}$ are the expansion factors between two adjacent cells in the rows $R_{n+1}$ to $R_{n}$ and in the columns $Z_{n+1}$ to $Z_{n}$, respectively. An illustration of the spatial grid around any node is provided in figure 2 .

The $\delta \tau$ in the dimensionless time grid is constant at the beginning of the chronoamperometry process where the change in flux is large. However, when the following criteria is achieved $\left(\tau_{s}\right)$, an exponential growth is applied for the dimensionless time step $\Delta \tau[26]$ :

$$
\frac{\Delta J}{\Delta \tau}<\left(\frac{\Delta J}{\Delta \tau}\right)_{\tau=0} \times 0.001
$$

Therefore:

$$
\begin{array}{ll}
\tau_{0}=0 & \\
\tau_{k+1}=\tau_{k}+\delta \tau & 0<\tau_{k} \leq \tau_{s} \\
\tau_{k+1}=\gamma_{\tau} \tau_{k} & \tau_{s}<\tau_{k}
\end{array}
$$

where $\gamma_{\tau}$ is the expansion factor between two adjacent time steps $(k+1$ and $k)$. The convergence criterion for the flux is defined to be:

$$
\left|\frac{J_{k+1}}{J_{k}}-1\right|<1 \times 10^{-9}
$$

The 2D mass transport system is solved using parallel computing of the alternating direction implicit (ADI) method. Each individual direction $\left(R_{n}, Z_{m}\right)$ in the ADI method is solved with the Thomas Algorithm for direct solving the using the Thomas algorithm. This allows the solution of the equations in a form of a tri-diagonal matrices to be obtained in $\mathrm{O}(\mathrm{n})$ operations. Numerical convergence study of the steady state flux with ensuring that the calculations 
are sufficiently accurate with the following values : $\delta R=1 \times 10^{-5}, \delta Z=1 \times$ $10^{-5}, \delta \tau=1 \times 10^{-9}, \gamma_{R}=1.1, \gamma_{Z}=1.3, \gamma_{\tau}=1.001$

Simulations were coded in $\mathrm{C}++$ with the OpenMP(Open Multi-programming) API for parallel programming. The simulations preformed on an Intel (R) Xeon (R) $3.2 \mathrm{Ghz}$, with approximately usage of 0.5GB RAM, and runtime between 5-20 minutes.

\section{Results and discussion}

The simulations are divided into three parts. We first report a case study of chronoamperometry at a simple microdisc electrode with an infinite insulating and non sticking surrounding surface. This allows validation of the code by examining the behaviour of the chronoamperometry curve, and comparing the values of the transient current with analytical and numerical results from previous works of Szabo [27] and Heinze [28]. Second in this section, we show the results of simulation for a fully sticking surface where the concentration is set to zero both on the microdisc electrode and on the surface (sheath). This simulation was made with different ratios of $R_{s d}$ to extract the relation of the shielding current to $R_{s d}$. These results for transport controlled sticking at the surrounding sheath can also be derived analytically to further validate the simulations. Third, results are discussed for intermediate sticking kinetics reflecting an intermediate case between a full sticking and non-sticking surrounding surface (sheath) where its controlled by finite adsorption kinetics $\left(k_{0}\right)$. This later study is made with representative ratios of $R_{s d}=10, R_{s d}=10^{2}$, and $R_{s d}=10^{3}$.

\subsection{Model Validation - simple microdisc electrode chronoam- perometry}

We use the model described above to generate the results of the dimensionless steady state current in a chronoamperometry process. The concentration at the disc electrode is set to zero. No sticking occurs on the sheath. Figure 3 shows the decay of the dimensionless flux at the microdisc as function of dimensionless time. The steady state flux is normalized to the theoretical value of the steady state flux at a microdisc electrode $(J / 4 C D R)$ and for large $\tau$ is converged to a satisfying value of 1.001. To assess the accuracy, we compare all the results with earlier simulation literature [28, 27], and also to the Shoup-Szabo approximate equation for chronoamperometry at a microdisc [27] (which is reported to be accurate to within $0.6 \%[27]$ ), where:

$$
\begin{gathered}
I=4 n F D c^{*} r_{d} f\left(\tau^{*}\right) \\
f\left(\tau^{*}\right)=0.7854+0.8862 \tau^{*-1 / 2}+0.2146 e^{-0.7823 \tau^{*-1 / 2}}
\end{gathered}
$$

For comparison purposes, we use $\tau^{*}=4 \tau$ to follow the Shoup-Szabo definition of the dimensionless time parameter. The variation of $f\left(\tau^{*}\right)$ with $\tau^{*}$ is presented in Table 2. The results show a good agreement with the simulation literature 
and the analytical approximation. It is worth mentioning that at short times the deviation from the other works shown here, can reach up to $0.4 \%$ but as time tends to large values, the deviation from the literature approaches zero. Overall this accuracy, validates the grid parameter chosen for the numerical 2D calculation of the chronoamperometry process and gives a reliable basis to for the further calculations reported in this work.

\subsection{The shielding effect - full sticking on the surrounding surface (sheath)}

Further simulation was next carried out to describe the shielding current on a microdisc electrode surrounded by a "full" sticking surface at when adsorption occurs at a mass transport controlled surface, so the concentration on both the surrounding insulating surface and on the electrode was set to zero. In this case, in the simulation we integrate the flux along the radius of the disc $\left(r_{d}\right)$ according to equation 17 . However, the sought result can also be obtained using the exact expression for the mass transport controlled flux distribution along the radius $[29,30,31]$ :

$$
j=\frac{2}{\pi} \frac{c^{*} D}{\sqrt{r_{s}^{2}-r^{2}}}
$$

By integrating the flux density from 0 to $R_{d}$ over the disc area, we can derive the shielded dimensionless flux as:

$$
J^{s}=2 \pi \int_{0}^{R_{d}} \frac{2}{\pi} \frac{1}{\sqrt{R_{s}^{2}-R^{2}}} R \mathrm{~d} R=-4\left(\sqrt{R_{s}^{2}-R_{d}^{2}}-R_{s}\right)
$$

Rearranging and approximating the root with a Taylor series, we get the relation of the shielding flux with $R_{d}$ and $R_{s}$ :

$$
J^{s}=4 R_{s}\left(\frac{R_{d}^{2}}{2 R_{s}^{2}}-\frac{R_{d}^{4}}{8 R_{s}^{4}}+\frac{R_{d}^{6}}{16 R_{s}^{6}}-\ldots\right) \approx \frac{2 R_{d}^{2}}{R_{s}}+O\left(\frac{R_{d}^{4}}{8 R_{s}^{3}}\right) \approx \frac{2 R_{d}^{2}}{R_{s}}
$$

Values of $J^{s}$ from the above relation and from simulation have been calculated for three different radius ratios, $R_{s d}$ and the results are summarized in Table 3 . Excellent agreement is evident. This results shows that the current shielded by a fully sticking surface can be simply generated by:

$$
I^{s}=2 F c^{*} D \frac{R_{d}^{2}}{R_{s}} \quad \text { for } R_{d}<<R_{s}
$$

In the following section we use this model to simulate the current shielded by a surface when adsorption occurs with finite absorption kinetics.

\subsection{Shielding effect - kinetically controlled adsorption}

Chronoamperometry simulation was first carried out for various adsorption rates at a representative ratio of $R_{s d}=1000$. Other ratios are discussed later in this 
section. This ratio of 1000 describes a typical case where a microelectrode of the order of $1 \mu \mathrm{m}$ is surrounded by an insulating surface of the order of $1 \mathrm{~mm}$ radius. The chronoamperometry curves are presented in figure 4 .

The results in figure 4 show the shielding effect on the flux/current $(J=$ $I / n F)$ when the surface is active toward adsorption. For low rates of adsorption, the curves have the typical profile of chronoamperometry on an unshielded microdisc, except for a slight deviation in the absolute values; close examination of the quasi-steady state flux absolute values shows that the dimensionless flux decreases by $7.5 \%$ from 4.02 for a non-sticking surface (the upper black thicker curve) to 3.71 for relatively low adsorption rate of $K=1 \times 10^{-4}$. It is important to note that this value is simulated for $R_{d}=1 \times 10^{-3}$. Therefore, in order to derive the dimensionless flux, we normalized the flux and $\mathrm{K}$ by $R_{d}$ to get the dimensionless form since the dimensionless $\mathrm{J}$ and $\mathrm{R}$ are defined using $r_{s}$ and $r_{d}$.

It can be seen that for intermediate rates of adsorption $(K=0.1-1000)$, the curves are characterized by two relaxations. The first is due to the disc and the second later one is related to the surrounding surface. This behaviour sharply increases with the adsorption rate constants over the range $10^{-3}<K / R_{d}<1$.

At very high adsorption rates $\left(K / R_{d}>1\right)$, the chronoamperometry profile suggests that the adsorption surface behaves as a full sticking surface and therefore the microdisc and the adsorbing surface can effectively be considered as a united surface, yielding one current relaxation on the electrode where the quasi-steady state flux value $\left(2 \times 10^{-6}\right)$ can also be derived from the analytical relation described earlier (equation 29). For the purposes of comparison, all the quasi-steady states values at different adsorbing rates and radius ratios are summarized in Table 4. Converting these values to dimensional currents using equation 18 can be very useful to determine the surrounding sheath activity and the shielded disc current by comparing these values with an experimental measured current at microdisc electrode.

Figure 5 compares the behaviour of the flux/current measured from the electrode with the ratio between the adsorbing surface and the electrode, where $R_{s d}=10,10^{2}$ and $10^{3}$. The profiles show that when the adsorbing surface becomes active, at higher $R_{s d}$ ratios the flux gets lower value and the relaxation time due to the adsorption surface becomes longer compared to larger $R_{s d}$ ratios. It can be seen that at $R_{s d}=10$ the relaxation time to the quasisteady state is fast and thus adsorption has a relatively smaller effect on the shape of the chronoamperometry curves. The profiles of the very low and high adsorption rates (figure 5a and figure 5c) particularly show the behaviour of a non-sticking and fully-sticking surface, and their absolute values (table 4) are in good agreement with the analytical expression described earlier.

In order to better understand the interaction between the adsorbing surface and the disc current, it is helpful to generate concentration profiles of the electrolyte/ adsorbing species in solution. Since the non-uniform diffusion flow generates different coverages across the insulating surface (sheath), it is necessary to choose $\Gamma$ to be artificiality large enough, so that $\theta<<1$ at all $\tau$. Consequently, the coverage is small enough to not affect the kinetics within the chronoamperometry process (since $(1-\theta) \approx 1$ ), allowing a quasi-steady 
state to be achieved. In order to determine the variation of adsorption uptake across the surface, we can examine the coverage rate $(d \theta / d \tau)$ along the surface $\left(R_{d}<R \leq R_{s}\right)$ as shown in figure 6 . The profiles of coverage rates also describes the flux profiles on the surface from equations 7 and 9 . These results (figure 6) show how the rate of coverage develops with time for the chronoamperometry process with a representative ratio of $R_{s d}=10$. Concentration maps in two dimensions are also provided in figure 7 to give a complementary view of the adsorption behavior. These concentrations maps were generated in two dimensions at quasi-steady state, near the electrode and the adsorption surface $(0 \leq R \leq 1,0 \leq Z \leq 0.6)$. At lower adsorbing rates of the surface (figure $6 \mathrm{a})$, we can see that the coverage is dominated mostly by the disc concentration gradient. Therefore, near the disc edge $\left(R_{d}\right)$ where the concentration is diluted, the flux and the coverage on the surface are lower, whereas on moving away from the disc radially towards the bulk solution, the concentration increases until it approaches the bulk concentration. In the same manner, the adsorption rate gradually increases along the surface. For intermediate rates (figure $6 \mathrm{~b}$ ), the surface becomes active, and thus higher rates of adsorption are observed at the edge of the surface typical for non-linear diffusion to the microelectrode surface [31]. Figure $6 \mathrm{c}$ and $7 \mathrm{~b}$ suggest that the surface almost fully controls the shape of the profile for the high adsorbing rate constant of $K=1000$. Moreover, figure 6 shows that a quasi-steady state is achieved for all the range of adsorbing rates as the gap between two consequent curves approaches to zero (the time grows exponentially with each curve). These quasi-steady state profiles give the coverage profiles that would be observed at a long chronoamperometry process, as long as the coverage fraction can be neglected $(\theta<<1)$.

Figure 8 shows the flux along the microdisc electrode. Since the boundary condition on the disc is assumed to be zero, the flux profile along the disc follows the behaviour of mass transport in a microdisc system. Comparing the values of the flux in the low and high adsorbing rates(figure 8a-b) suggests that the "edge effect" of high flux density in the edge of the microdisc, is moderated in high adsorbing rate as expected when the surface becomes fully active.

\section{Conclusions}

The simulations shown in this paper indicate that if significant adsorption occurs on the sheath used to insulate a microdisc electrode, then this can have a high impact on the measured current-time data because of significant 'shielding effect'. It was shown that this effect is reflected both in the characteristic shape of the relaxation in chronoamperometry and in the quasi-steady state current values. Moreover, the observed quasi-steady state currents are a function of the sheath to disc electrode radius ratio and the adsorption rate, and the

data reported can be directly used in experimental systems to determine this effect on the current responds measured at microdisc electrode surrounded by an insulating but adsorbing sheath. 


\section{Appendix}

In the main text, the adsorption rate is described using the rate constant $\mathrm{K}$. In the literature for the case of adsorbing nanoparticles, 'sticking coefficients' [22] are also used. For the purposes of comparison, we solve the simple case of an adsorbing surface with a hemispherical geometry.

We can relate the shielding current to the sticking coefficient which is a measure of the probability for species to stick on the surface[22] as follows:

$$
s=\frac{\text { Rate of species sticking to surface }}{\text { Rate of species collisions with surface }}
$$

The numerator in the above equation is described by $J_{s}$. However, in order to find the rate of collisions $\left(J_{c}\right)$ with the surface, we calculate the $Z$ flux towards the surface, where $Z$ approaches 0 from above:

$$
J_{c}=2 \pi \int_{R_{d}}^{R_{s}} \lim _{z \rightarrow 0^{+}}\left(\frac{\partial C}{\partial Z}\right) R \mathrm{~d} R
$$

The initial condition for the concentration is $C=1$ for all the space at $\tau=0$.

In this case, by considering a hemisphere which is uniformly adsorbing,the characteristic uniform diffusional flow towards the surface leads to uniform coverage. Therefore, when simulating only the surface without the electrode, the geometry can be reduced to a one dimension problem where the Fick's second law for sphere/hemisphere geometry becomes:

$$
\frac{\partial c}{\partial t}=D\left(\frac{\partial^{2} c}{\partial r^{2}}+\frac{2}{r} \frac{\partial c}{\partial r}\right)
$$

The boundary condition in the hemisphere is as follows:

$$
\begin{gathered}
\text { at } \quad \tau \geq 0 \quad D\left(\frac{\partial c}{\partial R}\right)_{r=0}=K_{0}(1-\theta) C_{R=0} \\
\text { at } \tau \geq 0 \text { and } R=R_{\max }, \quad C=C^{*}
\end{gathered}
$$

The coverage varies with time as described in the 2D model (equation 9).

Figure 9 shows the dimensionless steady state flux towards a hemisphere as function of the dimensionless rate constant, $K$. The normalized steady state flux describes the rate of the sticking species on the surface. At high rates, this normalized steady state flux approaches to unity as expected from the analytical solution for Fick's law:

$$
j(\tau)=\frac{1}{\sqrt{\pi \tau}}+\frac{1}{r_{s}}
$$

The flux at high rate also denotes the total rate of collisions with the surface. Therefore, this profile also represents the sticking coefficient for a uniform coverage under a diffusion driven flow. 


\section{Keywords}

micro-electrode, microdisc electrode, shielding, insulating sheath, adsorption, nanoparticle voltammetry

\section{References}

[1] C. Amatore, M. R. Deakin, R. M. Wightman, Journal of Electroanalytical Chemistry and Interfacial Electrochemistry 1987, 225, pp. 49-63.

[2] C. Amatore, N. Mota, C. Lemmer, C. Pebay, C. Sella, L. Thouin, Journal of Electroanalytical Chemistry 2008, 80, pp. 9483-9490.

[3] M. R. Deakin, R. M. Wightman, C. Amatore, Journal of Electroanalytical Chemistry and Interfacial Electrochemistry 1986, 215, pp. 49-61.

[4] A. M. Bond, M. Fleischmann, J. Robinson, Journal of Electroanalytical Chemistry and Interfacial Electrochemistry 1984, 168, pp. 299-312.

[5] C. Amatore, S. Arbault, I. Bonifas, M. Guille, Biophysical Chemistry 2009, 143(3), pp. 124-131.

[6] C. Amatore, S. Arbault, Y. Bouret, M. Guille, F. Lematre, ChemPhysChem 2010, 11(13), pp. 2931-2941.

[7] M. V. Mirkin, F. R. Fan, A. J. Bard, Journal of Electroanalytical Chemistry 1992, 328, pp. 47-62.

[8] R. W. Murray, Chemical Reviews 2008, 108(7), pp. 2688-2720.

[9] N. V. Rees, Y. G. Zhou, and R. G. Compton, RSC Adv. 2012, 2, pp. 379-384.

[10] A. M. Bond, Analyst 1994, 119, pp. 1R-21R.

[11] K. Tschulik, C. Batchelor-McAuley, H. Toh, E. J. E. Stuart, R. G. Compton, Phys. Chem. Chem. Phys. 2014, 16, pp. 616-623.

[12] Y. Zhou, N. V. Rees, R. G. Compton, Angewandte Chemie International Edition 2011, 50(18), pp. 4219-4221.

[13] E. O. Barnes, R. G. Compton, Journal of Electroanalytical Chemistry 2013, 693, pp. 73-78.

[14] K. R. Ward, N. S. Lawrence, R. S. Hartshorne, R. G. Compton, Journal of Electroanalytical Chemistry 2012, 683, pp. 37-42.

[15] I. J. Cutress, R. G. Compton, Chemical Physics Letters 2011, 508, pp. 306313.

[16] H. Brenner. Chemical Engineering Science 1961, 16(3), pp. 242-251. 
[17] I. J. Cutress, E. Dickinson, R. G. Compton, Journal of Electroanalytical Chemistry 2011, 655(1), pp. 1-8.

[18] I. Streeter, R. G. Compton, The Journal of Physical Chemistry C 2007, 111(49), pp. 18049-18054.

[19] J. Crank, The mathematics of diffusion 1979, Oxford university press.

[20] R. G. Compton, K. L. Pritchard, J. Chem. Soc., Faraday Trans. 1990, 86(1), pp. 129-136.

[21] I. Langmuir, Journal of the American Chemical Society 1918, 40(9), pp. 1361-1403.

[22] Y. Zhou, N. V. Rees, R. G. Compton, Chemical Physics Letters 2011, 514(4), pp. 291-293.

[23] A. Einstein, Annalen der Physik 1905, 322(8), pp. 549-560.

[24] D. Gavaghan, Journal of Electroanalytical Chemistry 1998, 456(1), pp. 1323.

[25] J. Crank, P. Nicolson, Mathematical Proceedings of the Cambridge Philosophical Society 1947, 43, pp. 50-67.

[26] D. J. Gavaghan, Journal of Electroanalytical Chemistry 1997, 420, pp. 147-158.

[27] D. Shoup, A. Szabo, Journal of Electroanalytical Chemistry and Interfacial Electrochemistry 1982, 140(2), pp. 237-245.

[28] J. Heinze, Journal of Electroanalytical Chemistry and Interfacial Electrochemistry 1981, 124(1), pp. 73-86.

[29] Y. Saito, Review of Polarography 1968, 15(6), pp. 177-187.

[30] M. A. Bender, H. A. Stone, Journal of Electroanalytical Chemistry 1993, 351 , pp. 29-55.

[31] R. G Compton, C. E Banks, Understanding voltammetry 2006, Imperial College Press. 


\begin{tabular}{lll}
\hline Parameter & Description & Units \\
\hline$A$ & Electrode area & $\mathrm{m}^{2}$ \\
$r_{d}$ & Electrode radius & $\mathrm{m}$ \\
$r_{s}$ & Surface radius & $\mathrm{m}$ \\
$D$ & Diffusion coefficient & $\mathrm{m}^{2} \mathrm{~s}^{-1}$ \\
$c$ & Concentration & $\mathrm{mol} / \mathrm{m}^{3}$ \\
$c^{*}$ & Bulk solution concentration & $\mathrm{mol} / \mathrm{m}^{3}$ \\
$\theta$ & Fractional surface coverage & \\
$\Gamma$ & Surface coverage of a full monolayer & $\mathrm{mol} / \mathrm{cm}^{2}$ \\
$k^{0}$ & Adsorption rate constant & $\mathrm{ms}$ \\
$t$ & Time & $\mathrm{s}$ \\
$j^{D}$ & Flux per unit area & $\mathrm{mol} /\left(\mathrm{m}^{2} s\right)$ \\
$J^{D}$ & Net Flux & $\mathrm{mol} / \mathrm{s}$ \\
$j$ & Dimensionless flux density & \\
$J$ & Dimensionless net flux & \\
$C$ & $c / c^{*}$ & \\
$R$ & $r / r_{s}$ & \\
$R_{d}$ & $r_{d} / r_{s}$ & \\
$R_{s}$ & 1 & \\
$Z$ & $z / r_{s}$ & \\
$\tau$ & $D t / r_{s}^{2}$ & \\
$K$ & $k_{0} r / D$ & \\
$\gamma$ & $\Gamma k^{0} /\left(c^{*} D\right)$ & \\
\hline
\end{tabular}

Table 1: Dimensional and dimensionless parameters 


\begin{tabular}{lllll}
\hline$\tau^{*}=4 \times \tau$ & $f\left(\tau^{*}\right)$ & & & \\
\hline & This work & Heinze [28] (simulation) & Shoup and Szabo [27] (simulation) & Shoup and Szabo \\
\hline 0.01 & 9.677 & 9.66 & 9.632 & 9.647 \\
0.04 & 5.246 & 5.237 & 5.226 & 5.221 \\
0.09 & 3.777 & 3.772 & 3.768 & 3.755 \\
0.25 & 2.612 & 2.609 & 2.602 & 2603 \\
0.64 & 1.969 & 1.969 & 1.969 & 2.008 \\
1.21 & 1.689 & 1.688 & 1.68 & 1.695 \\
2.25 & 1.496 & 1.495 & 1.491 & 1.496 \\
4.00 & 1.367 & 1.367 & 1.366 & 1.374 \\
6.76 & 1.280 & 1.28 & 1.279 & 1.285 \\
\hline
\end{tabular}

Table 2: Validation of the chronoamperometric currents at different dimensionless times 


\begin{tabular}{lllll}
\hline$R_{d}$ & $R_{s d}$ & $J^{s}($ Simulation $)$ & $J^{s}($ Analytical expression) & J without sticking surface (Theoretical, $J=4$ \\
\hline 0.1 & 10 & $1.99 \times 10^{-2}$ & $2.0 \times 10^{-2}$ & 0.4 \\
0.01 & 100 & $1.98 \times 10^{-4}$ & $2.0 \times 10^{-4}$ & 0.04 \\
0.001 & 1000 & $2.00 \times 10^{-6}$ & $2.0 \times 10^{-6}$ & 0.004 \\
\hline
\end{tabular}

Table 3: Numerical and analytical values of the dimensionless shielding flux at various radius ratios 


\begin{tabular}{llll}
\hline$K / R_{d}$ & $R_{s d}=10$ & $R_{s d}=100$ & $R_{s d}=1000$ \\
\hline $1 \times 10^{-5}$ & 4.013 & 4.015 & 3.985 \\
$1 \times 10^{-4}$ & 4.010 & 3.972 & 3.707 \\
0.001 & 3.983 & 3.664 & 2.143 \\
0.01 & 3.733 & 2.022 & 0.385 \\
0.1 & 2.312 & 0.343 & 0.0521 \\
1 & 0.619 & 0.0621 & 0.0109 \\
10 & 0.263 & 0.0274 & 0.00481 \\
100 & 0.211 & 0.0213 & 0.00311 \\
1000 & 0.203 & 0.0204 & 0.00227 \\
$1 \times 10^{4}$ & 0.202 & 0.0202 & 0.00206 \\
$1 \times 10^{5}$ & 0.201 & 0.0201 & 0.00203 \\
\hline
\end{tabular}

Table 4: Quasi-steady state values at various $R_{s d}$ and dimensionless $\mathrm{K}$. 


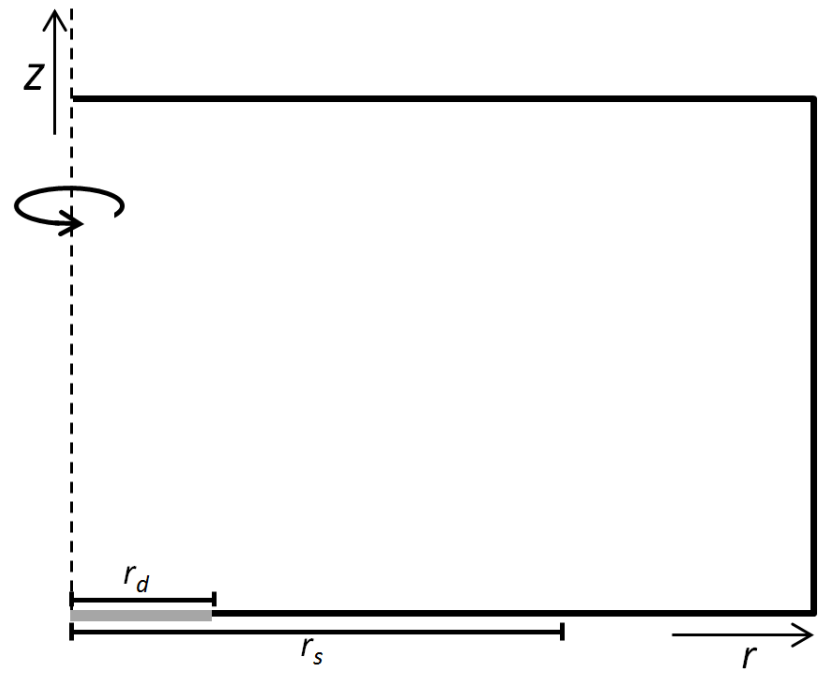

Figure 1: Schematic of the 2D calculation area of the micro-disc electrode with radius $r_{d}$ and the adsorbing surface with radius $r_{s}$. 


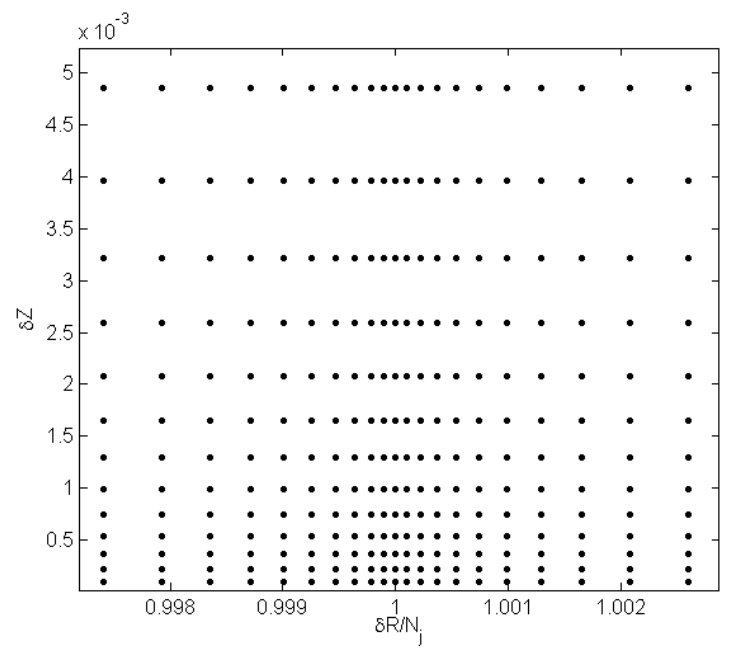

Figure 2: The discretization around the nodes in the spatial grid. 


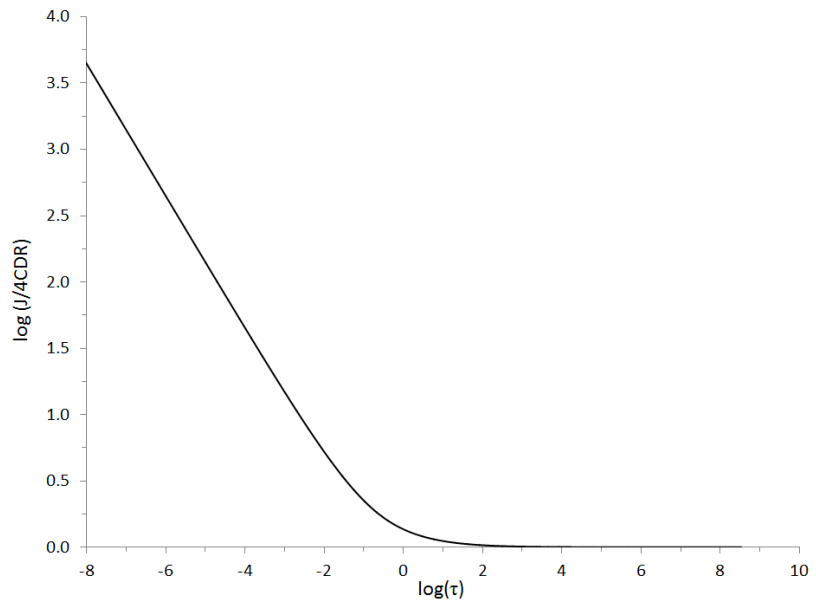

Figure 3: Simulation of CA process in a microdisc electrode. The curve is presented in logarithmic scales. 


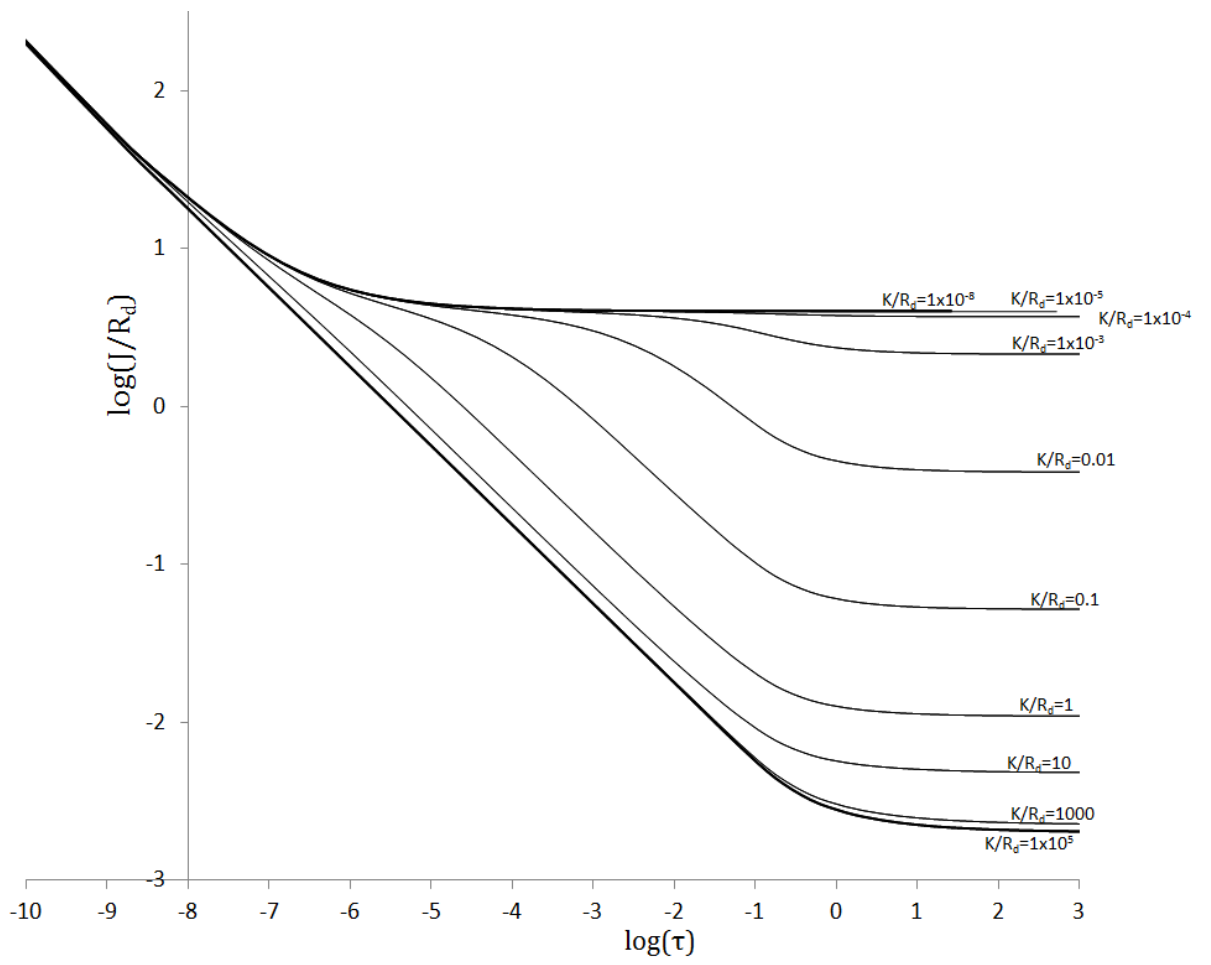

Figure 4: Chronoamperometry curves at the total flux simulated on the microdisc surrounded by adsorbing surface with a wide range of $\mathrm{K}$ (from $1 \times 10^{-8}$ to $\left.1 \times 10^{5}\right)$. 
a)

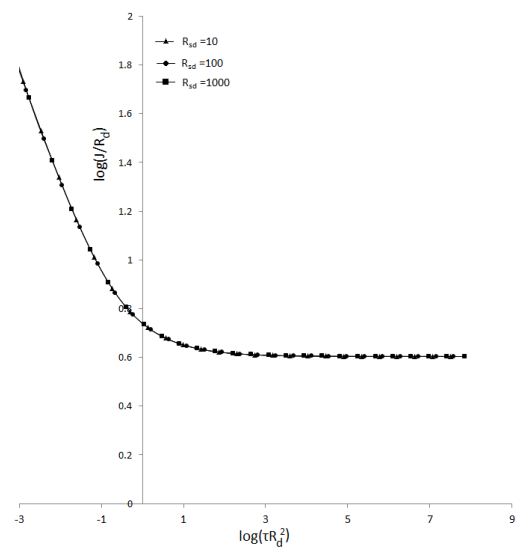

b)

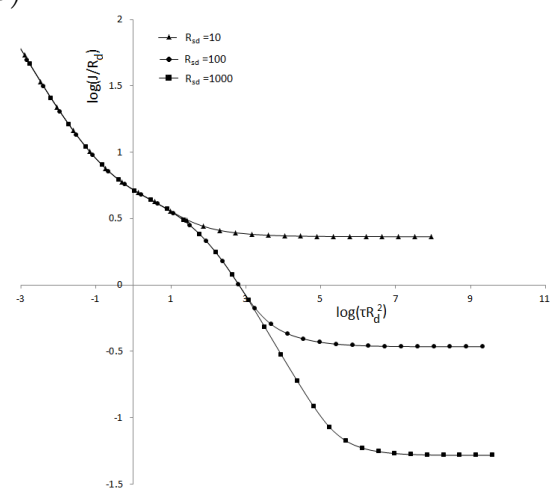

c)

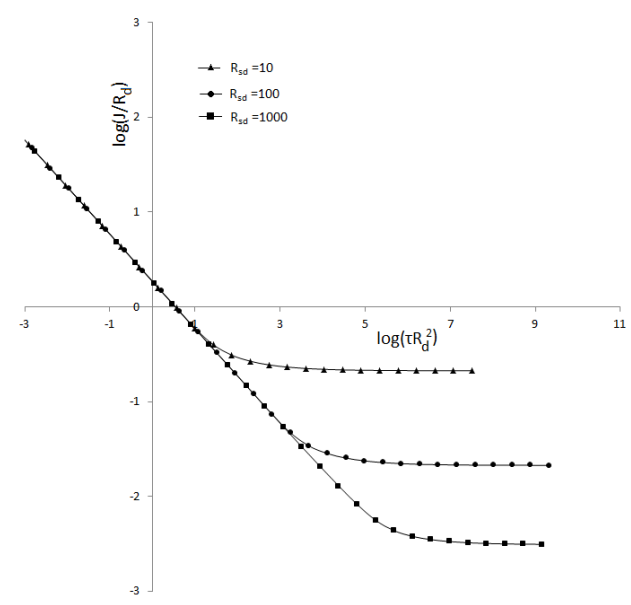

Figure 5: A comparison of chronøamperometry curves in different $R_{s d}$ $(1000,100,10)$ at (a) $K / R_{d}=1 \times 10^{-4}$, (b) $K / R_{d}=0.1$, and (c) $K / R_{d}=100$. In order to compare between the results of the chronoamperometry curves for different $R_{s d}$, we normalize the flux, $\tau$ and $K$ relative to the calculated $R_{d}$. 

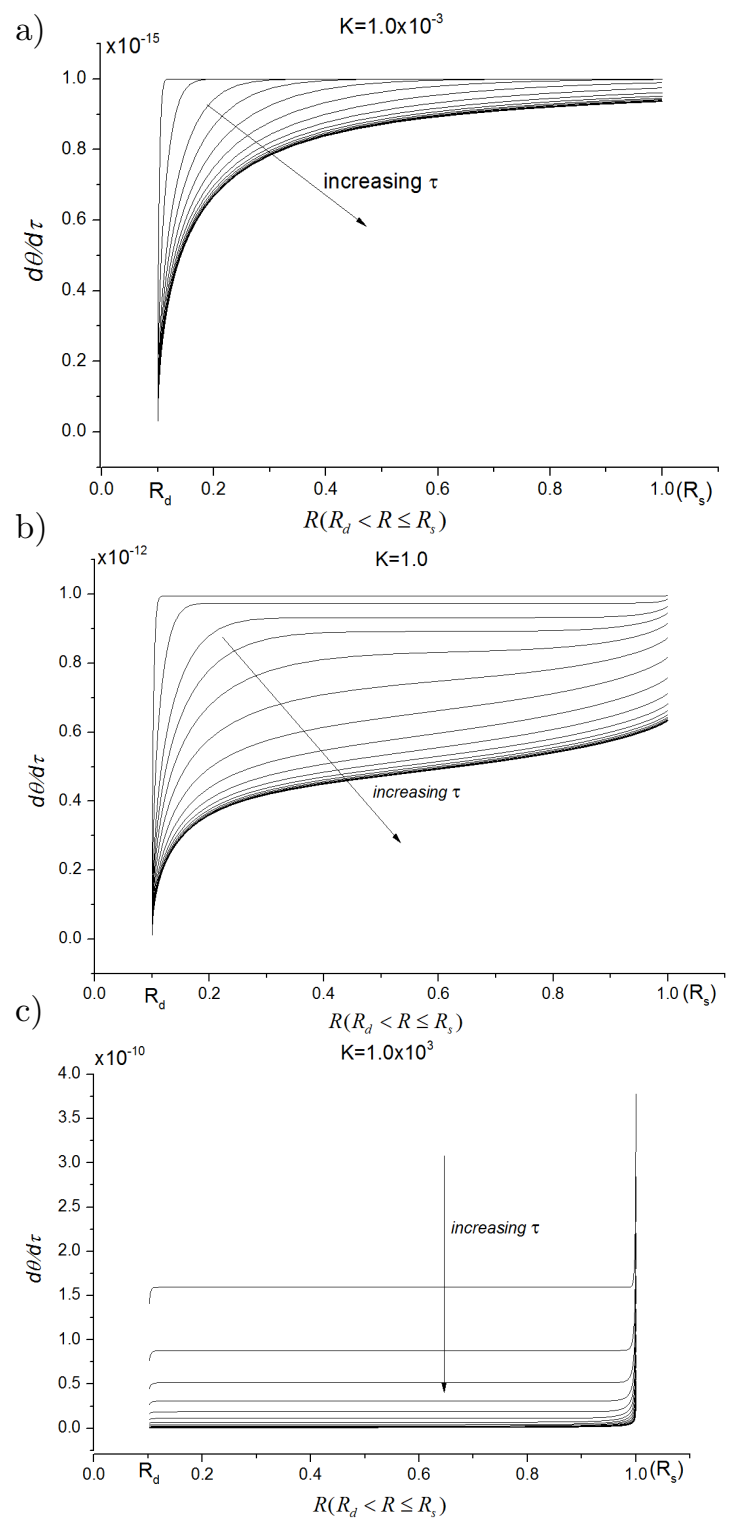

Figure 6: The rate of coverage $(d \theta / d \tau)$ along the surface $\left(R_{s d}=10\right)$ at various $\tau$ in three different adsorbing coefficient rates: $K=10^{-3}(\mathrm{a}), K=1.0(\mathrm{~b})$, and $K=10^{3}(\mathrm{c})$. The $\tau$ values from the upper to the downer curves are: $1.6 \times 10^{-6}, 6.0 \times 10^{-6}, 1.80 \times 10^{-5}, 5.05 \times 10^{-5}, 1.39 \times 10^{-4}, 3.79 \times 10^{-4}, 1.03 \times 10^{-3}, 2.80 \times 10^{-3}, 7.62 \times 10^{-3}, 2.07 \times 10^{-}$ and the thick line is $\tau=9099$. 
a)
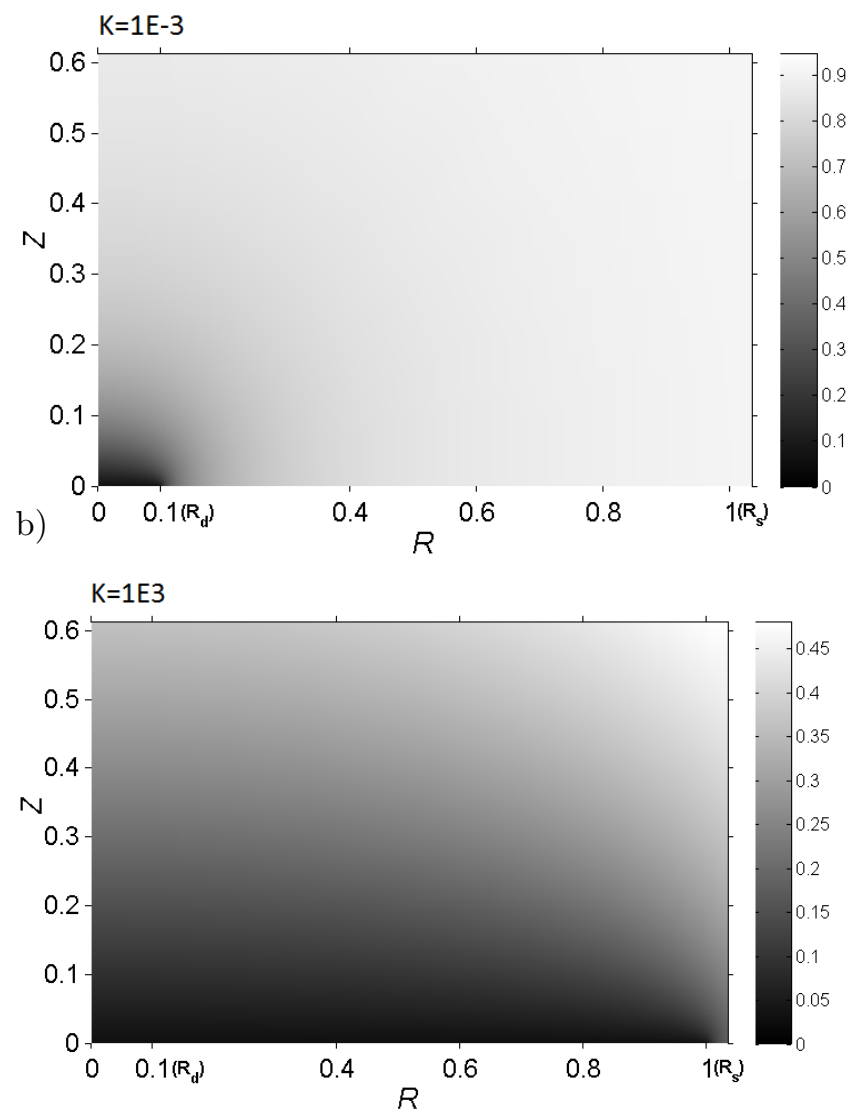

Figure 7: Concentration maps of the electrode and surface area $\left(R_{s d}=10\right)$ in low adsorbing rate, $K=0.001$ (a) and high adsorbing rate, $K=1000$ (b) 

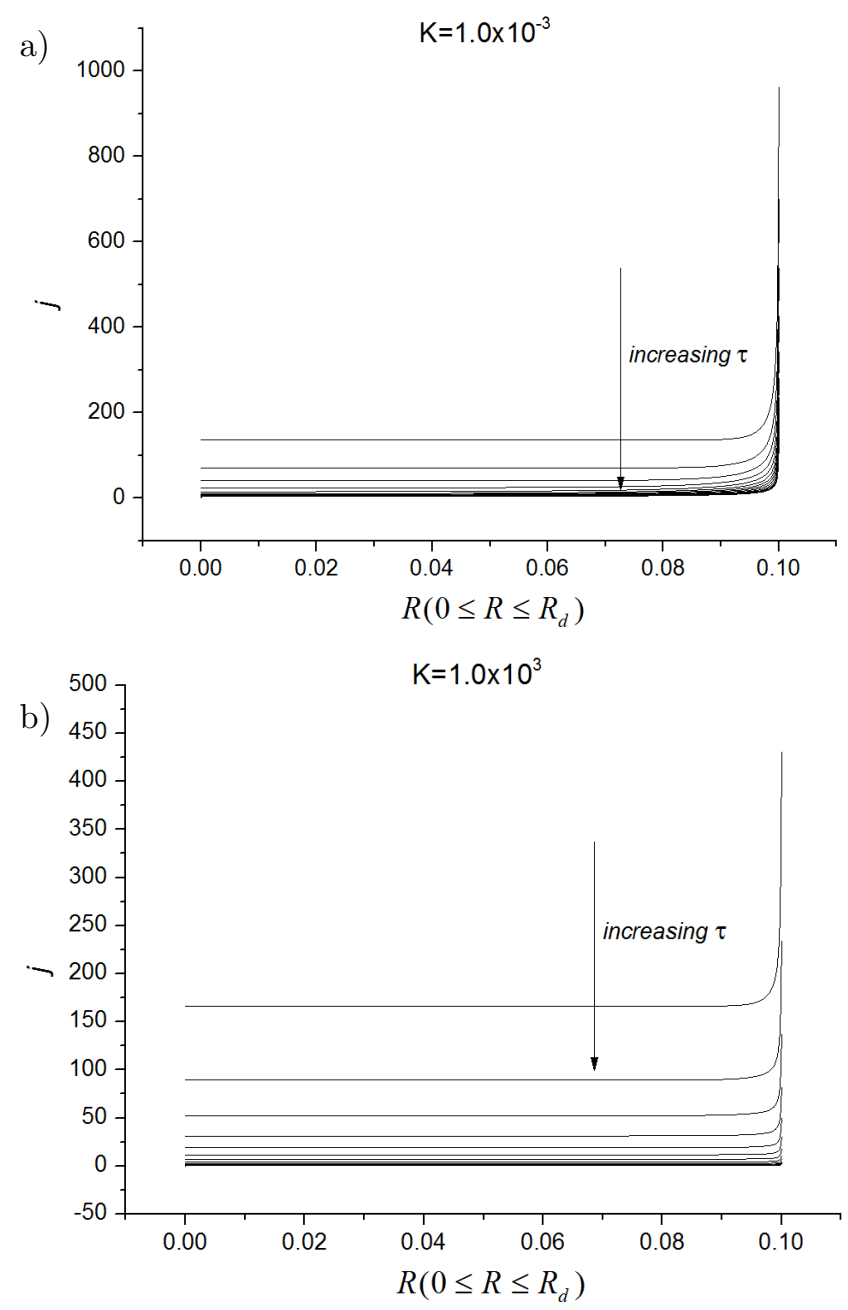

Figure 8: The flux density $(j)$ inward the disc at various $\tau$ in two adsorbing coefficient rates: $K=10^{-3}(\mathrm{a})$ and $K=10^{3}(\mathrm{~b})$, $R_{s d}=10$. The $\tau$ values from the upper to the downer curves are: $1.6 \times 10^{-6}, 6.0 \times 10^{-6}, 1.80 \times 10^{-5}, 5.05 \times 10^{-5}, 1.39 \times 10^{-4}, 3.79 \times 10^{-4}, 1.03 \times 10^{-3}, 2.80 \times 10^{-3}, 7.62 \times 10^{-3}, 2.07 \times 10^{-}$ and the thick line is $\tau=9099$. 


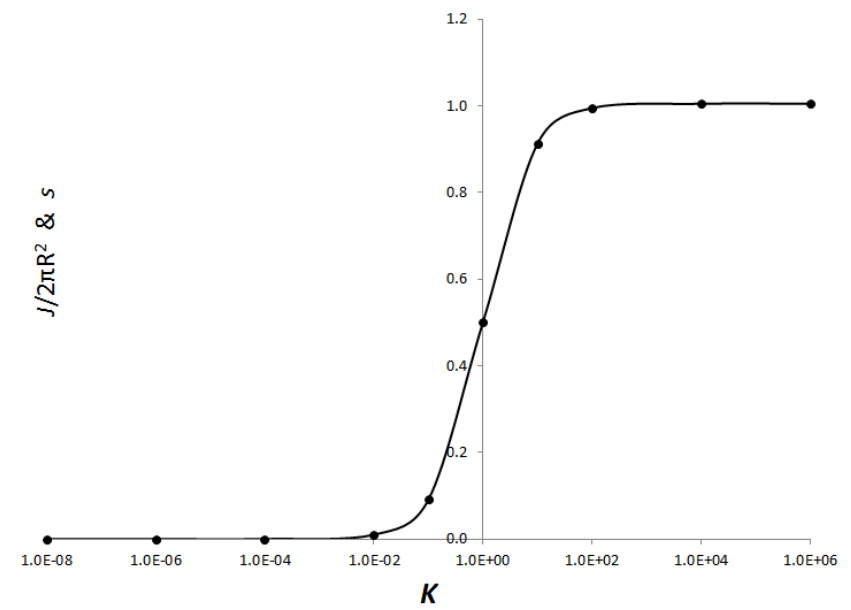

Figure 9: Profile of the flux and the sticking coefficient as function of the kinetic rate in hemisphere surface . 